\title{
Malaria Diagnostic Capacity of Private Health Facilities in Ethiopia
}

\author{
Mesele DamteArgaw* \\ Private Health Sector Program, P.O. Box 2372 code 1250, Addis Ababa, Ethiopia
}

*Corresponding Author: Mesele Damte Argaw, Private Health Sector Program, P.O. Box 2372 code

1250, Addis Ababa, Ethiopia, Email: mdamte5@gmail.com

\begin{abstract}
Background: Prompt and accurate diagnosis of malaria is a part of effective disease management strategies.The aim of this survey was to assess the malaria diagnosis capacity of private health facilities before engaging into public private partnership for malaria care services in Ethiopia.
\end{abstract}

Methods: This facility-based cross-sectional survey was conducted from January through September 2012. The data were obtained using multiple sources, which includes interview of facility owners or managers, facility observation using checklist, and record review using data abstraction form. The data were entered and analysed using Microsoft Office 2010 and SPSS IBM version 20, respectively.

Results: In this survey we enrolled 185 private health facilities with a response rate $97.3 \%$. Almost all private health facilities have access to electric power supply (180/185; 97.3\%), close to half had access to running piped water $(101 / 185 ; 51.8 \%)$ and all dedicated some places for patient waiting area. Regarding with professional mix, slightly lower than half $(520 / 1165 ; 44.6 \%)$ of the health service providers were nurses followed by one fourth laboratory professionals (289/1165; 24.8\%).Almost all (149/151; 98.6\%) of private health facilities has at least a single functional binocular microscope. At the time of the survey half $(5 / 9$; $55.5 \%)$ of hospital, (6/54;11.1\%) of Higher Clinic and (6/90; 6.6\%)of Medium Clinic laboratories have absolute methanol stock on hand which is necessary to perform thin blood film. During the survey time slightly higher than two third $(121 / 185 ; 65.4 \%)$ of private facilities have placed sharp proof containers in a desirable location. The majority $(153 / 185 ; 82.7 \%)$ of the private health facilities use microscope to diagnosis malaria, while less than one fifth $(34 / 185 ; 16.4 \%)$ facilities use Rapid Diagnostic Tests (RDTs). The record review revealed that each facility were served for 116, 101, 27 and 26 malaria suspected, laboratory investigated, confirmed and presumed malaria diagnosed cases with in the previous two weeks, respectively. All facility owners were willing and committed to work in public private partnership for malaria care services.

Conclusions: The assessed private health facilities were equipped with the necessary amenities useful to ensure patient comfort and staffed with different mix of professionals. Almost all private health facilities have basic equipment and supplies but few of them have the capacity to perform both thin and thick blood film for parasitological confirmation of malaria. This baseline assessment was served as an input in identifying and intervening in a planned manner to improve the capacity of private health facilities.

Keywords: Capacity, Diagnosis, Ethiopia, Malaria, Private Health Facilities.

\section{INTRODUCTION}

Malaria is one of the most severe public health problems worldwide, exerting an unacceptable toll on the health and economic welfare of the world's poor communities [1]. According to World Health Organization (WHO) latest estimates, 198 million cases of malaria occurred globally in 2013 (uncertainty range 124-283 million) and in the same year the disease led to 584000 deaths (uncertainty range 367 000-755
000). The burden is heaviest in the WHO African Region, where an estimated $90 \%$ of all malaria deaths occur, and in children aged under 5 years, who account for $78 \%$ of all deaths [2].

Prompt and accurate diagnosis of malaria is part of effective disease management strategy [3]. It also plays as an entry point to ensure the quality of malaria case management both by health care providers and patient [4]. Many countries adopted the WHO recommendation on universal 
parasitological confirmation by microscopy or alternative by rapid diagnostic test [3] in all patients suspected of malaria before treatment is started.

According to the report of Ethiopian Federal Ministry of Health [EFMOH] National Health Account V, about $19.8 \%$ of Ethiopian outpatient received service from private for profit health facilities [5].Similarly, Jima et al (2010) confirm that more than one quarter of mothers sought medical care for their feverish child first from formal private health providers [6]. Deressa et al. (2009) reported that in Oromia Region of Ethiopia, more than $60.0 \%$ mothers with recent episode of malaria received initial treatment from non -public sector [7].

Brugha and Zwi (1998) affirm that the service offered by private providers often perceived by users to be more attractive [8]. However, evidences suggest that serious deficiencies in technical quality are often present. Khin et al (2015) affirms the global malaria control program was challenged by the occurrence of drug resistance malaria infection [9]. Among many contributing factors for emergence of drug resistance plasmodium strains, the unregulated with the formal and informal private health sector blamed for poor adherence of accepted recommendations [10].

According to WHO (2007) health system consists of all organizations, people and actions whose primary intent is to promote, restore or maintain health. This includes efforts to influence determinants of health as well as more direct health-improving activities. A health system is therefore more than the pyramid of publicly owned facilities that deliver personal health services, and includes state and non-state actors such as non-governmental organizations, civil society organizations, and the private sector [11].

The current health system of Ethiopia organized into three-tier health care delivery approaches: Primary level is one covering about 60,000100,000 people; level two is a General Hospital covering 1-1.5 million people; and level three is a comprehensive Specialized Hospital for about 3.5-5 million people. Furthermore, the rapidly growing numbers of private for profit and not for profit organizations are expected to work in partnership to boost health service coverage and utilization [12].
The WHO health systems framework consists of six building blocks, namely, service delivery, health workforce; health information; medical technologies (including medical products, vaccines, and other technologies); health financing; leadership; and governance [11]. In implementing the last building block, leadership and governance, the United State Agency for International Development (USAID) funded Private Sector Project (PSP) to pilot on Public Private Partnership Mix Direct Observed Short Course (PPM DOTS) for Tuberculosis Management. The terminal evaluation of the PPM DOTS program conducted by Emmett and Whalen affirm the feasibility of the implemented Tuberculosis (TB) and Human Immune deficiency Virus (HIV) Programs in Public Private Mix model [13].Following the above evaluation, the EFMOH decided to scale up PPM DOTS Services from 20 up to 181 facilities [14]. Since 2009, United States Agency for International Development /Ethiopia funded Public Private Partnership for PPMDOTS, TB/HIV, ART, PMTCT, FP/STIs and Malaria Programs.

Before integrating malaria care service in selected Public Private Partnership (PPP) facilities with one of the major public health important disease or services ( i.e. TB , HIV/AIDS, Family Planning programs), PHSP in collaboration with six Regional State Health Bureaus conducted service availability, facility readiness and willingness assessment. The aim of this survey was to assess the malaria diagnosis capacity of private health facilities before engaging in public private partnership for malaria care services. The second objective of the survey was to extract information about the performance of facilities providing malaria care services.

\section{Materials And Methods}

\subsection{Study Setting and Survey}

The survey was conducted in five Regional States and one City Administration of Federal Democratic Republic of Ethiopia, namely Amhara, Oromia, Tigray, Southern Nation Nationalities and Peoples [SNNP] and Hareri Regions and Dire Dawa City Administration (Figure 1).

The targeted districts were selected in consultation with respective Regional Health 
Bureaus (RHBs). The surveyed health facilities include 9 hospitals, 54 Higher Clinics, 90 Medium Clinics and 32 Lower linics. We collected facility based data in two phases. The first phase collected in three Regions between January and February 2012. And the second phase in in the rest regions between August and September 2012.

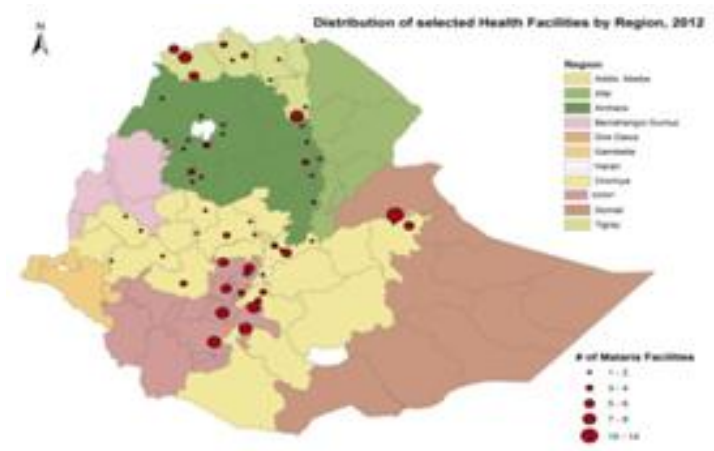

Figure1. Location and distribution of surveyed health facilities in Ethiopia

*This figure clearly depicted the distribution of selected health facilities in six regional states of Ethiopia.

A pretested standardized structured questionnaire was adopted for this survey [15]. The tools comprised structured questionnaires for interviews, checklist used for observation and data extraction forms for record review. Major categories of the collected data were the following: i. general information about the health facility, ii. Resent Staff profile of facility i.e. human resource capacity /\# health workers, malaria diagnosis and treatment training, iv. Facility infrastructure and availability of laboratory equipment and supplies, v. availability of national treatment guidelines, Standard Operating Procedures (SOPs), Job aids, WHO malaria microscopy bench aid, accessing supportive supervision, historical information about reporting and partnership vi. Malaria case load, patient information from malaria morbidity and mortality and comprehensive Laboratory registers, Vii. Health facility's willingness and commitment to share and shoulder in national responsibility for providing standard malaria services

Table1. Premises at 185 survey private health facilities September 2012

\begin{tabular}{|l|l|l|l|l|l|l|l|l|}
\hline \multirow{2}{*}{ Profession } & \multicolumn{2}{|c|}{ Hospital } & \multicolumn{2}{c|}{ Higher Clinic } & \multicolumn{2}{c|}{ Medium clinic } & \multicolumn{2}{c|}{ Lower Clinic } \\
\cline { 2 - 10 } & Freq & \% & Freq & \% & Freq & \% & Freq & \% \\
\hline Amenities & & & & & & & & \\
\hline Electric Power & 9 & $100.0 \%$ & 54 & $100.0 \%$ & 89 & $98.8 \%$ & 28 & $87.5 \%$ \\
\hline Running Water & 9 & $100.0 \%$ & 54 & $100.0 \%$ & 14 & $33.3 \%$ & 24 & $75.0 \%$ \\
\hline Waiting area & 9 & $100.0 \%$ & 54 & $100.0 \%$ & 90 & $100.0 \%$ & 32 & $100.0 \%$ \\
\hline
\end{tabular}

Six assessment teams comprising members of the Private Health Sector Program (PHSP), delegates of RHBs and district health office disease prevention and control experts conducted the data collection. Prior to deployment the data collectors received a day long briefing and exercises on the survey tool.

\subsection{Methods of Measurements and Procedures}

This facility-based cross-sectional survey was conducted from January through September 2012. The data were obtained using multiple sources, which includes one on one interview with head or owners of the facilities, checklist for observation and patient information abstraction form as record review.

\subsection{Data Processing and Analysis}

The data were checked for completeness and cleaned manually. The data were entered into Microsoft Office Excel 2010 and analyzed using Statistical Package for Social Science (SPSS) research (SPSS IBM version 20).

\section{Results}

In this survey we enrolled 185 private health facilities with a response rate 97.3\%. Five facilities were dropped due to incomplete information or missing key informant after frequent facility visit. Of the 185 selected facilities $9(4.8 \%)$ were general hospitals, 54(29.2\%) were Higher Clinics, 90(48.6\%) were medium Clinics and the rest $32(17.3 \%)$ were Lower Clinics.

\subsection{Amenities}

Table 1 depicted amenities of selected private health facilities. All hospitals and higher clinics have access to 24 hours electric power and piped water supplies. Almost all private health facilities dedicated some place for patient waiting area. To maintain patient comfort, all hospitals, majority $94.4 \%$ of higher clinics and 92.2\% of medium clinics have television and/or video show services. However, the availability of IEC/BCC materials ranges from $33.3 \%$ for hospitals to zero for lower clinics. 


\begin{tabular}{|l|l|l|l|l|l|l|l|l|}
\hline TV or video shows & 9 & $100.0 \%$ & 51 & $94.4 \%$ & 74 & $92.2 \%$ & 14 & $43.7 \%$ \\
\hline IEC/ BCC materials & 3 & $33.3 \%$ & 32 & $59.2 \%$ & 34 & $37.7 \%$ & 0 & $0.0 \%$ \\
\hline Toilet facility & 9 & $100.0 \%$ & 54 & $100.0 \%$ & 90 & $100.0 \%$ & 32 & $100.0 \%$ \\
\hline Surveyed facility & 9 & $100.0 \%$ & 54 & $100.0 \%$ & 90 & $100.0 \%$ & 32 & $100.0 \%$ \\
\hline PHSP supported sites & 9 & $100.0 \%$ & 44 & $81.4 \%$ & 35 & $38.8 \%$ & 0 & $0.0 \%$ \\
\hline
\end{tabular}

The median number of beds per hospital was 38 (range: 24- 185). The median number of beds per Higher Clinic was eight (range: 3 - 88). And the median number of beds per Medium Clinic was 2 (range: 1- 10). Only one Lower Clinic, workplace health facility, has five beds.

Out of 185 surveyed facilities the majority 174 $(94.0 \%)$ were private for profit, $6(3.2 \%)$ private not for the profit and the rest $5(2.7 \%)$ were workplace private health facilities. Half of these facilities 91(49.2\%) have been working with Regional State Health Bureaus through the implementation of PPM DOTS program, Human Immune Deficiency Virus (HIV) or Family Planning services. Almost all 180 $(97.3 \%)$ of the facilities have access to 24 hours electric power and piped water supplies.

\subsection{Clinical And Laboratory Staff Profile}

In Ethiopia availability of qualified regular staff is a requirement to run private health facilities set by Ministry of Health. In the surveyed facility there were 85 Specialist (all types), of which $31(36.5 \%)$ were working in Private Hospitals, 48(56.2\%) were working in Higher Clinics. Slight higher than half $50(51.5 \%)$ of the General Practitioners were working in Higher Clinics. Three fourth 57 (75.0\%) of Public Health Officers were working in Medium Clinics. One third 149 (28.7\%) of nurses were working in hospitals. The majority 125 (43.3\%) of laboratory staffs were working in Medium Clinics. Moreover, only one third of laboratory professionals participated a five days malaria diagnosis training (Table 2).

Table2. Distribution of Clinical and Laboratory staff in 185 surveyed private health facilities, September 2012

\begin{tabular}{|c|c|c|c|c|c|c|c|c|c|}
\hline \multirow[t]{2}{*}{ Profession } & \multicolumn{2}{|c|}{ Hospital } & \multicolumn{2}{|c|}{ Higher Clinic } & \multicolumn{2}{|c|}{ Medium clinic } & \multicolumn{2}{|c|}{ Lower Clinic } & \multirow[t]{2}{*}{ Total } \\
\hline & Freq & $\%$ & Freq & $\%$ & Freq & $\%$ & Freq & $\%$ & \\
\hline \multicolumn{10}{|l|}{ Clinical Staffs } \\
\hline MD specialist (all type) & 31 & $36.5 \%$ & 48 & $56.2 \%$ & 6 & $7.0 \%$ & 0 & $0.0 \%$ & 85 \\
\hline General Practitioner & 12 & $12.4 \%$ & 50 & $51.5 \%$ & 35 & $35.8 \%$ & 0 & $0.0 \%$ & 97 \\
\hline Public Health Officer & 0 & $0.0 \%$ & 18 & $23.7 \%$ & 57 & $75.0 \%$ & 1 & $1.3 \%$ & 76 \\
\hline BSc. Nurse & 21 & $37.5 \%$ & 19 & $33.7 \%$ & 15 & $26.4 \%$ & 1 & $1.8 \%$ & 56 \\
\hline Diploma Nurse & 149 & $28.7 \%$ & 201 & $38.6 \%$ & 134 & $25.7 \%$ & 36 & $6.9 \%$ & 520 \\
\hline Junior Nurse & 2 & $4.8 \%$ & 12 & $28.6 \%$ & 14 & $33.3 \%$ & 14 & $33.3 \%$ & 42 \\
\hline Laboratory Personnel & 37 & $12.8 \%$ & 121 & $41.9 \%$ & 125 & $43.3 \%$ & 6 & $2.1 \%$ & 289 \\
\hline Lab personnel trained on malaria diagnosis* & 13 & $35.1 \%$ & 44 & $36.6 \%$ & 32 & 25.6 & 0 & 0.0 & 89 \\
\hline
\end{tabular}

NB: Denominator all lab personnel

\subsection{Essential Laboratory Equipment And Supplies}

Table3. Availability of Malaria Diagnostic equipment at surveyed facilities September 2012

\begin{tabular}{|l|l|l|l|l|l|l|}
\hline \multirow{2}{*}{ Item Description } & \multicolumn{2}{l}{ Hospital } & \multicolumn{2}{l|}{ Higher Clinic } & \multicolumn{2}{l|}{ Medium Clinic } \\
\cline { 2 - 7 } & Freq & $\%$ & Freq & $\%$ & Freq & $\%$ \\
\hline Functional Binocular Microscope & 9 & $100.0 \%$ & 53 & $98.1 \%$ & 89 & $98.8 \%$ \\
\hline Oil Immersion & 9 & $100.0 \%$ & 54 & $100.0 \%$ & 89 & $98.8 \%$ \\
\hline Lens paper & 4 & $44.4 \%$ & 18 & $33.3 \%$ & 17 & $18.8 \%$ \\
\hline Slide staining jar (Coplin staining Jar) & 4 & $44.4 \%$ & 21 & $38.8 \%$ & 18 & $20.0 \%$ \\
\hline Slide staining rack & 8 & $88.9 \%$ & 46 & $85.2 \%$ & 82 & $91.1 \%$ \\
\hline Slide drying rack & 4 & $44.4 \%$ & 4 & $7.4 \%$ & 3 & $3.3 \%$ \\
\hline Slid Box of 100 & 9 & $100.0 \%$ & 43 & $79.6 \%$ & 50 & $55.5 \%$ \\
\hline Microscope slide & 9 & $100.0 \%$ & 54 & $100.0 \%$ & 90 & $100.0 \%$ \\
\hline Frosted end slide & 3 & $33.3 \%$ & 34 & $63.0 \%$ & 40 & $44.4 \%$ \\
\hline Lead Pensile & 3 & $33.3 \%$ & 32 & $59.3 \%$ & 38 & $42.2 \%$ \\
\hline Funnel & 3 & $33.3 \%$ & 25 & $46.3 \%$ & 16 & $17.7 \%$ \\
\hline Measuring cylinder & 3 & $33.3 \%$ & 21 & $38.9 \%$ & 15 & $16.6 \%$ \\
\hline Beaker/ volumetric flask & 6 & $66.6 \%$ & 8 & $14.8 \%$ & 2 & $6.6 \%$ \\
\hline Bunsen Burner & 3 & $33.3 \%$ & 33 & $61.1 \%$ & 32 & $35.5 \%$ \\
\hline Forceps & 9 & 100.05 & 54 & $100.0 \%$ & 90 & $100.0 \%$ \\
\hline
\end{tabular}




\begin{tabular}{|l|l|l|l|l|l|l|}
\hline Lancet & 9 & $100.0 \%$ & 54 & $100.0 \%$ & 90 & $100.0 \%$ \\
\hline Thermometer & 9 & $100.0 \%$ & 35 & $64.8 \%$ & 51 & $56.6 \%$ \\
\hline Digital Timer & 7 & $77.8 \%$ & 41 & $75.9 \%$ & 48 & $53.3 \%$ \\
\hline Cell Counter (Tally or differential) & 6 & $66.7 \%$ & 35 & $64.8 \%$ & 52 & $57.7 \%$ \\
\hline Brown Bottle of 2lt & 5 & $55.6 \%$ & 29 & $53.7 \%$ & 23 & $25.5 \%$ \\
\hline Giemsa Stain (3\% or 10\%) Stock solution & 8 & $88.9 \%$ & 53 & $98.1 \%$ & 89 & $98.8 \%$ \\
\hline Wright Stain & 7 & $77.8 \%$ & 36 & $66.7 \%$ & 30 & $33.3 \%$ \\
\hline Absolute Methanol & 5 & $55.5 \%$ & 6 & $11.1 \%$ & 6 & $6.6 \%$ \\
\hline Gylcerol & 5 & $55.5 \%$ & 6 & $11.1 \%$ & 6 & $6.6 \%$ \\
\hline Filter Paper (Whatman \#1) & 3 & $33.3 \%$ & 21 & $38.9 \%$ & 18 & $20.0 \%$ \\
\hline Glove & 9 & $100.0 \%$ & 54 & $100.0 \%$ & 90 & $100.0 \%$ \\
\hline Cotton wool & 9 & $100.0 \%$ & 54 & $100.0 \%$ & 90 & $100.0 \%$ \\
\hline
\end{tabular}

Almost all hospitals (100.0\%), higher clinics $(98.1 \%)$, medium clinic $(98.8 \%)$ and only two $(6.2 \%)$ of lower clinics owned at least one functional binocular electrical microscope. Consumables like gloves, cotton and lancet. Close to half of the facilities do have slide box of 100 . One third of hospitals $(33.3 \%)$ have frosted end slides, lead pencils, funnels and measuring cylinder. But higher clinics better equipped with $63.0 \%$ forested end slides, $59.0 \%$ lead pencils, $46.0 \%$ funnels and $38.9 \%$ measuring cylinder. The proportion of laboratories which has cell counter (tally or differential counter) ranges from $57.7 \%$ or medium clinics to $66.7 \%$ hospitals. Almost all facilities have Giemsa stain (stock solution $3.0 \%$ ) stock on hand but one hospital, one higher clinic and one medium clinic doesn't have it. Wright stain was found in $77.8 \%$ of hospitals a, $75.9 \%$ of higher clinics and $57.7 \%$ of medium clinics. At the time of the survey, half $55.5 \%$ of hospital, $11.1 \%$ of Higher Clinics and $6.6 \%$ of Medium clinics laboratories have absolute methanol stock on hand (Table 3)No facilities were found with Disodium hydrogen phosphate (anhydrous) $\left(\mathrm{Na}_{2} \mathrm{HPO}_{4}\right)$, Sodium di- hydrogen phosphate hydrate $\left(\mathrm{NaH}_{2} \mathrm{PO}_{4} \mathrm{H}_{2} \mathrm{O}\right)$, and Glass Beads $(3.0 \mathrm{~mm})$. There were no facilities found using Leishman's stain or Field Stain Solution for malaria diagnosis.

\subsection{Working Documents and Health Information Management}

The national malaria treatment guidelines $\left(2^{\text {nd }}\right.$ Ed) were found in $6(66.7 \%)$ of hospitals and 6 $(11.1 \%)$ of higher clinics. Malaria microscopy bench aid which was developed by WHO, observed in $2(22.2 \%)$ of Hospitals and 4 (7.4\%) of Higher Clinics. The availability of comprehensive laboratory registers varies from $9(100.0 \%)$ in hospitals to $31.1 \%$ in medium clinics. No lower clinics found with patient morbidity or laboratory registers. The majority $77.8 \%$ of hospitals, a little lower than half 24 (44.4\%) of Higher Clinics and one third 28 $(31.1 \%)$ of Medium Clinics used to submit standard reports to respective Woreda (District) Health Office son weekly bases. The majority $88.9 \%$ of hospitals and slightly higher than half $53.7 \%$ of higher clinics received joint supportive supervision in the previous six months (Table 4).

Table4. Distribution of National Treatment Guidelines, Job aids, and SOP, September 2015

\begin{tabular}{|l|l|l|l|l|l|l|l|l|}
\hline \multirow{2}{*}{ Item Description } & \multicolumn{2}{l|}{ Hospital } & \multicolumn{2}{l|}{ Higher Clinic } & \multicolumn{2}{l|}{ Medium Clinic } & \multicolumn{2}{l|}{ Lower Clinic } \\
\cline { 2 - 9 } & Freq & $\%$ & Freq & $\%$ & Freq & $\%$ & Freq & $\%$ \\
\hline National Malaria Treatment Guidelines & 6 & $66.7 \%$ & 6 & $11.1 \%$ & 12 & $13.3 \%$ & 3 & $9.4 \%$ \\
\hline SOP for malaria Microscopy & 4 & $44.4 \%$ & 7 & $12.9 \%$ & 8 & $8.8 \%$ & 0 & $0.0 \%$ \\
\hline Malaria microscopy bench aid & 2 & $22.2 \%$ & 4 & $7.4 \%$ & 0 & $0.0 \%$ & 0 & $0.0 \%$ \\
\hline Job aid malaria RDT procedure & 0 & $0.0 \%$ & 1 & $1.9 \%$ & 0 & $0.0 \%$ & 0 & $0.0 \%$ \\
\hline Comprehensive Lab log book & 9 & $100.0 \%$ & 24 & $44.4 \%$ & 28 & $31.1 \%$ & 0 & $0.0 \%$ \\
\hline Weekly malaria report (THO) & 7 & $77.8 \%$ & 24 & $44.4 \%$ & 28 & $31.1 \%$ & 0 & $0.0 \%$ \\
\hline Supportive Supervision & 8 & $88.9 \%$ & 29 & $53.7 \%$ & 29 & $32.2 \%$ & 3 & $9.4 \%$ \\
\hline Number of Facility Surveyed & 9 & $100.0 \%$ & 54 & $100.0 \%$ & 90 & $100.0 \%$ & 32 & $100.0 \%$ \\
\hline
\end{tabular}

\subsection{Malaria Suspect, Presumed and Confirmed Cases}

Table5. Employed malaria diagnosis methods, and quality assurance scheme, September 2015

\begin{tabular}{|l|l|l|l|l|l|l|l|l|}
\hline \multirow{2}{*}{ Profession } & \multicolumn{2}{|c|}{ Hospital } & \multicolumn{2}{c|}{ Higher Clinic } & \multicolumn{2}{c|}{ Medium clinic } & \multicolumn{2}{c|}{ Lower Clinic } \\
\cline { 2 - 10 } & Freq & $\%$ & Freq & $\%$ & Freq & $\%$ & Freq & $\%$ \\
\hline Malaria Diagnosis & & & & & & & & \\
\hline Microscopy (Blood film) & 9 & $100.0 \%$ & 54 & $100.0 \%$ & 89 & $98.8 \%$ & 2 & $6.2 \%$ \\
\hline
\end{tabular}

ARC Journal of Public Health and Community Medicine 


\begin{tabular}{|l|l|l|l|l|l|l|l|l|}
\hline Malaria RDTs* & 6 & $66.7 \%$ & 6 & $11.1 \%$ & 13 & $14.4 \%$ & 7 & $21.8 \%$ \\
\hline Internal Quality Control & 9 & $100.0 \%$ & 43 & $79.6 \%$ & 35 & $38.8 \%$ & 0 & $0.0 \%$ \\
\hline External Quality Control & 0 & $0.0 \%$ & 0 & $0.0 \%$ & 0 & $0.0 \%$ & 0 & $0.0 \%$ \\
\hline
\end{tabular}

NB: *Malaria Rapid Diagnostic Tests

At the time of the survey almost all facilities were providing malaria diagnosis and treatment services. Malaria microscopy test were done in all hospitals higher clinics and medium clinics. Two lower clinics were observed while diagnosing malaria using blood film. Moreover, $6(66.7 \%)$ of hospitals, $6(11.1 \%)$ of higher clinics, $13(14.4 \%)$ of medium clinics and 7 (21.8\%) of lower clinics used RDTs to diagnose malaria. In the surveyed facilities documents were observed in all hospitals and $38.8 \%$ of medium clinics for Internal Quality Control (IQC). But no facilities were found as a part of External Quality Control system (Table 5).
Record reviews were made to measure the malaria case load in each facility for the previous two weeks duration. The mean malaria suspect was $116.3 \pm 171.9$. The larger proportion of malaria suspect cases visited higher clinics with mean $133.5 \pm 186$. The average lab services for two weeks duration was $101.0 \pm 159.2$. During the same period an average $26.4 \pm 54.3$ patients per facility were treated on presumed diagnosis and an equivalent number of malaria confirmed cases $27.3 \pm 44.8$ were treated (Table 6).

Table6. Average malaria case load, laboratory investigation, presumed and confirmed malaria diagnosis September 2012

\begin{tabular}{|l|l|l|l|l|l|l|l|l|}
\hline \multirow{2}{*}{ Level of facility } & \multicolumn{2}{|l|}{ Malaria Suspect } & \multicolumn{2}{l|}{ Lab Investigated } & \multicolumn{2}{l|}{ Presumed Diagnosis } & \multicolumn{2}{l|}{ Confirmed Diagnosis } \\
\cline { 2 - 10 } & Mean & SD & Mean & SD & Mean & SD & Mean & SD \\
\hline Hospital & 81.4 & 144.4 & 75.4 & 145.0 & 5.6 & 11.9 & 12.6 & 27.9 \\
\hline Higher Clinic & 133.5 & 186.3 & 119.6 & 151.7 & 39.5 & 76.7 & 30.4 & 55.2 \\
\hline Medium Clinic & 119.3 & 175.1 & 101.5 & 172.9 & 20.9 & 39.0 & 27.6 & 39.5 \\
\hline Lower Clinic* & 35.3 & 32.5 & 32.0 & 49.2 & 23.9 & 29.2 & 21.3 & 40 \\
\hline Over all & 116.3 & 171.9 & 101.0 & 159.2 & 26.4 & 54.3 & 27.3 & 44.8 \\
\hline
\end{tabular}

NB: SD: Standard Deviation, * Lab investigated \& Confirmed

\subsection{Biosafety (Infection prevention)}

Table 7 presented the level of biosafety practices in laboratories of the surveyed facilities. All laboratories in nine hospital, followed by 46 (85\%) located in higher clinics prepared sharp proof container with desirable locations. We observed that all laboratory staffs wore protective coat/gown during service hours. We observed use of protective gloves during phlebotomy in 5 (55.5\%) of hospitals, 20(37.0\% of higher clinic and $18(20.0 \%)$ of medium clinics. The highest hand washing facilities were observed in hospitals $(100.0 \%)$, followed by higher clinic13 $(24.1 \%)$. But slightly higher than half of the higher clinics $(53.7 \%)$ prepared antiseptics hand rub. Only a little higher than one third $(40.7 \%)$ of the labs in higher clinics and close to one fifth of medium clinics prepared decontamination solution (Chlorine $0.5 \%$ ) every 24 hours (Figure 2 ).

Table7. Utilization of personal protection Equipment, hand hygiene and waste management, September 2012

\begin{tabular}{|c|c|c|c|c|c|c|c|c|}
\hline \multirow[t]{2}{*}{ Item Description } & \multicolumn{2}{|c|}{ Hospital } & \multicolumn{2}{|c|}{ Higher Clinic } & \multicolumn{2}{|c|}{$\begin{array}{l}\text { Medium } \\
\text { Clinic }\end{array}$} & \multicolumn{2}{|c|}{ Lower Clinic } \\
\hline & Freq & $\%$ & $\begin{array}{l}\text { Fre } \\
\mathrm{q}\end{array}$ & $\%$ & Freq & $\%$ & $\begin{array}{l}\mathrm{Fr} \\
\mathrm{eq}\end{array}$ & $\%$ \\
\hline Wear glove during phlebotomy & 5 & $55.5 \%$ & 20 & $37.0 \%$ & 18 & $20.0 \%$ & 1 & $3.1 \%$ \\
\hline Wear gown/coat & 9 & $100.0 \%$ & 54 & $100.0 \%$ & 90 & $100.0 \%$ & 32 & $100.0 \%$ \\
\hline Hand washing facility & 9 & $100.0 \%$ & 13 & $24.1 \%$ & 9 & $10.0 \%$ & 2 & $6.3 \%$ \\
\hline Antiseptics for hand rub & 9 & $100.0 \%$ & 29 & $53.7 \%$ & 27 & $30.0 \%$ & 1 & $3.1 \%$ \\
\hline Disinfectants/decontamination & 9 & $100.0 \%$ & 22 & $40.7 \%$ & 15 & $16.6 \%$ & 5 & $15.6 \%$ \\
\hline $\begin{array}{l}\text { Sharp proof waste } \\
\text { Container (biohazard) }\end{array}$ & 9 & $100.0 \%$ & 46 & $85.2 \%$ & 62 & $68.8 \%$ & 4 & $12.5 \%$ \\
\hline Waste segregation & 3 & $33.3 \%$ & 33 & $61.1 \%$ & 29 & $32.0 \%$ & 0 & $0.0 \%$ \\
\hline Incinerator & 9 & 100.0 & 51 & $94.4 \%$ & 83 & $92.2 \%$ & 23 & $71.8 \%$ \\
\hline
\end{tabular}




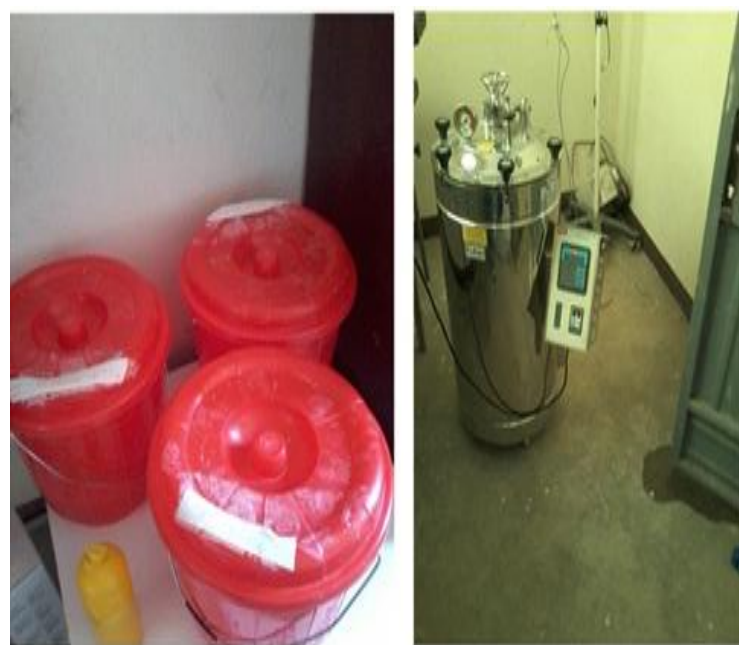

Figure2. Good Infection Prevention practices: three bucket solution for proper decontamination procedure and functioning autoclave (Photo by: Argaw, M)

*The figures shows the good practices with regards to infection prevention and instrument processing in selected private facilities.

One third $(33.3 \%)$ of hospitals, two third $(61.1 \%)$ of higher clinics and one third $(32.0 \%)$ of medium clinics practiced proper segregation of medical waste (Figure3).

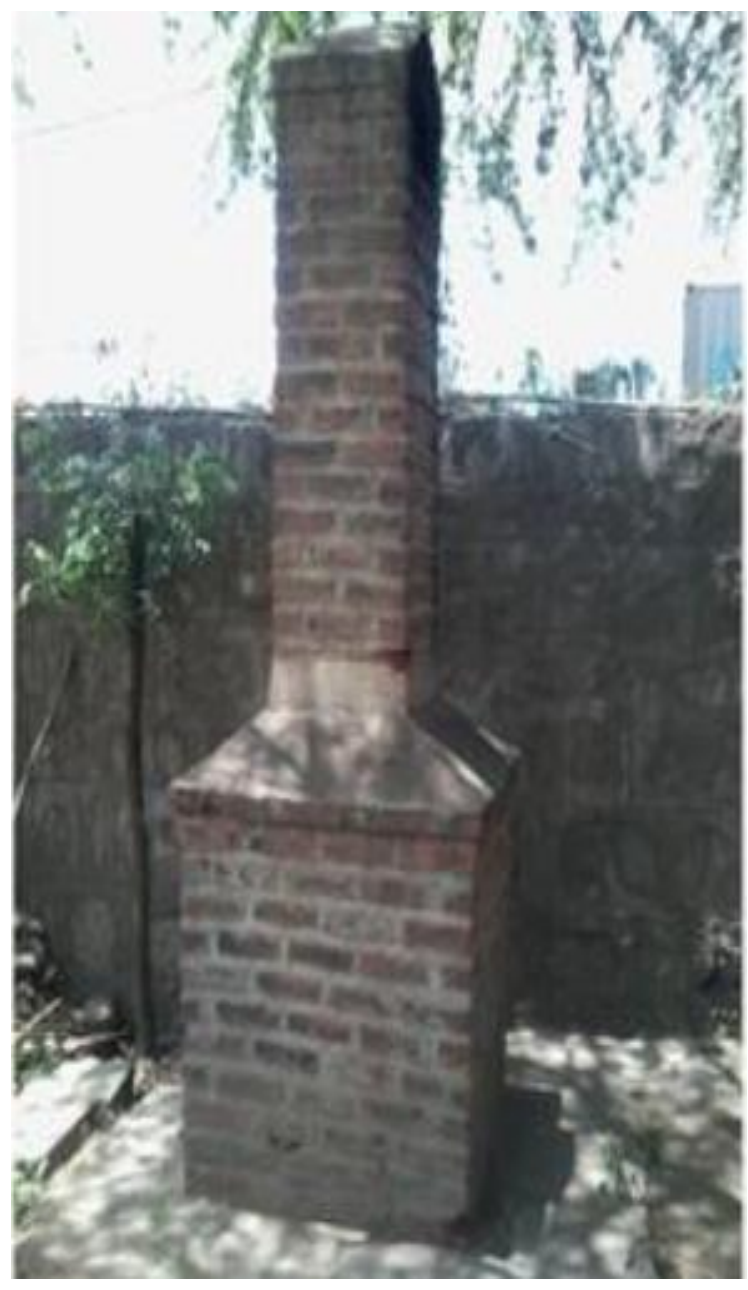

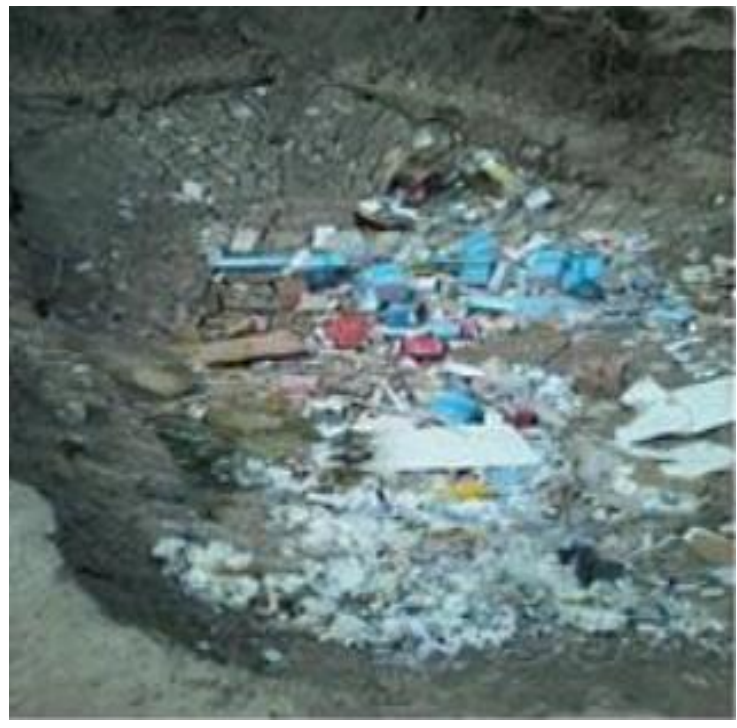

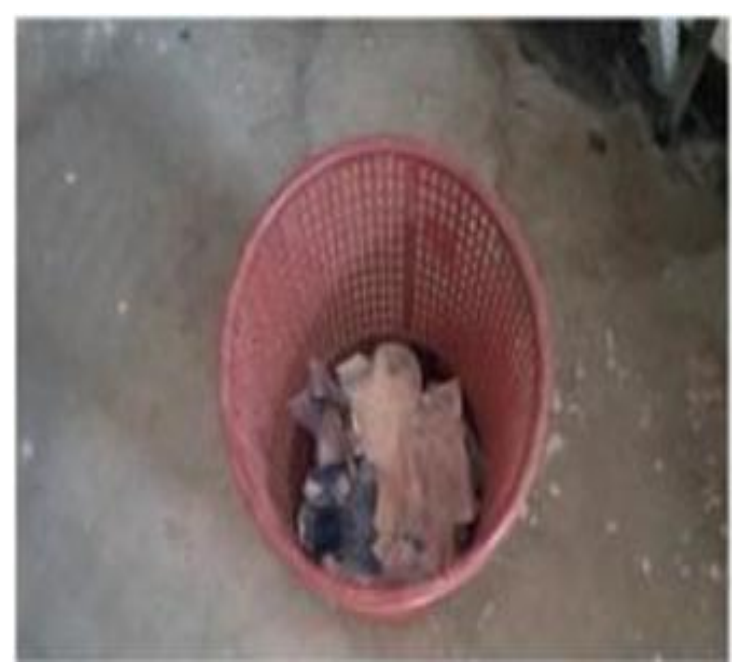

Figure3. Waste Management: functioning incinerator, disposal of sharps in shallow pit and mix of sharps and biohazards with dry wastes (Photo by: Argaw M)

*The figure represents waste management practice, a model facility with clean and functional incinerator, a facility with waste disposal in shallow ditch and mixed management of biohazard and dry wastes.

\subsection{Willingness and Commitment}

Almost all 185 assessed facilities were willing to work in public private mix partnership approach on malaria care services. They are willing to adhere with the reporting and recording requirement of the public health system. They are ready to ensure quality of services though practicing Internal Quality Control (IQC) and participating in External Quality Assurance (EQA) scheme. The owners expressed their readiness to work in partnership through cost sharing for orientations and trainings. Moreover, they were ready to provide malaria care service at subsidized cost using only consultation fee. 


\section{DISCUSSIONS}

This malaria diagnostic capacity assessment was conducted in five regional states and one city administration of Ethiopia. Data were collected from 185 private health facilities located in malaria endemic areas. In this survey we enrolled 9 Hospitals, 54 Higher Clinics, 90 Medium Clinics and 32 Lower Clinics. There are few published reports of malaria diagnostic capacity of Public health facilities in Oromia Region of Ethiopia \& Indonesia. [16][17][18][19]. While this assessment report address the malaria diagnostic capacity of Private health facilities in Ethiopia a.

Most of the assessed private health facilities were found clean and have sound patient waiting area. However, small number of facilities offered health messages to their clients. Almost all facilities, except few Lower Clinics have access to tap water and 24 hours electric power supplies. But interruption of power supplies were reported as a challenge for ensuring provision of malaria microscopy services as per the national recommendations. This finding was in agreement with Abreha et al. (2014) reported public health facilities suffered from interruption of electric power [18].

The staffing patter of the assessed facilities was found to the level of the national recommendations for each level. This could be happed due to the existing regulation and standards necessary for renewal of licensure. Rao et al. reported the comparable number of staff working in private sector as a moonlighter [14], i.e those who work in the public facility during the day time might work in private facility during the night. But the average number of laboratory professionals working in surveyed facilities significantly reduced from 4.1 personnel's per hospital to 1.4 personnel's per medium clinic. This shortage of human resource could be the main reason for provision of presumed malaria diagnosis and treatment. One third of lab personnel received malaria laboratory diagnosis training in the previous one year. This finding is a little higher than only $6 \%$ of facilities reported at least one laboratory personnel received training in the last 12 months. This finding could be due to that slightly lower than half of the facilities $(47.6 \%)$ received eight days comprehensive TB/HIV and malaria trainings [14].

We assessed the level of practice with regards to biosafety. And almost all staff had worn coat/gown during working hours. The overall biosafety assessment was found poor. Less than half of the laboratory personnel's worn protective gloves during phlebotomy and dispose biohazard wastes without segregation. This finding was found much higher risk than the public health laboratory assessment $75 \%$ of facilities used sharp poor materials[14] [17].

Almost all (98.8\%) of the assessed facilities has at least one functional binocular microscope. But less than half of the facility has Coplin staining jar, drying rack and Cell Counter (tally or differential counter). Moreover, few of the assessed facilities have essential equipment or supplies to prepare Giema stock solution with in their laboratory [20].

Preforming thin and tick blood film in routine test is recommended [1][4][20]. With the supplies on hand only $55.5 \%$ of hospitals, $11.1 \%$ of higher clinics and $6.6 \%$ of medium clinics have the necessary capacity to perform thin blood film. This finding is much lower than $28.0 \%$ of public facilities including research laboratories and malaria centers were performing thick and thin blood film in Oromia Region [14].

We observed the presence of National Malaria Guidelines $\left(2^{\text {nd }} \mathrm{Ed}\right)$ in $14.5 \%$ of surveyed facilities[21] i.e 6 in hospitals, 6 in higher clinics and 12 in medium clinics and 3 in Lower Clinics. This finding was in line with the report of Jerene et al in Oromia region only 4 (5\%) private health facilities received national malaria guidelines form public sector [22]. This finding was in much lower than $31 \%$ of public facility does have national malaria guidelines in Oromia Region. And In Tanzania using nationwide service availability survey less than one third of six hundred facilities had national malaria treatment guidelines [23]. In this survey we observed few malaria job aids, Standard Operating Procedures and bench aids. This finding show the effort to improve the quality of malaria diagnosis was very low. However, one third $(31.8 \%)$ of the facilities reported that they received joint supportive supervision from public sector.

Almost all facilities except lower clinics provide malaria laboratory diagnostic services using blood film. Half $(47.0 \%)$ of the surveyed facilities had documented their level of effort to ensure quality of lab service they provided in the form of Internal Quality Control (IQC). But no facility was found to be the part of External Quality Assessment (EQA) scheme. Moreover, 
slightly lower than one fifth $(17.3 \%)$ of the facilities were observed while performing malaria Rapid Diagnostic Test. This is in line with Jerene et al found $16(18 \%)$ of private health facilities were using RDT in four zone of Oromia region [22]. This could be due to high case load per lab personnel or Health workers expect much reliable and valid report using RDT than Microscopy.

The average malaria suspected cases were 116 patients in two weeks duration. Though Higher Clinics were seeing the largest number of patients significant number of patients were visiting Lower clinics. In all facilities half of malaria patients were treated based on confirmed parasitological diagnosis. This finding was in line with previous studies in which approximately one half of malaria patients received correct treatment [24][25][26].

Almost all private health facilities were willing to work in partnership with the public sector. Their level of commitment was found high. Most private health facilities are willing to provide complete care for malaria services only with consultation and lab service fee. They are willing to invest on staff training, improving lab services and to participate in EQA scheme.

\section{Conclusions}

The assessed private health facilities were equipped with amenities useful for patient comfort and staffed with different mix of health professionals. But the level of biosafety for patients and health professionals were found poor. Almost all surveyed private health facilities have essential laboratory equipment and supplies. However, few of them have the capacity to prepare Giemsa Stock solution within their premises. In addition, only a few facilities were able to perform thin and thick blood film as per the national recommendation. Despite large number of patients is seeking medical care for malaria care services from private health facilities, the diagnostic capacity were challenged by lack of essential equipment's, supplies and technical support. This baseline assessment helps Private Health Sector Program (PHSP) to identify major intervention areas and to evaluate the PPP for malaria program after planned intervention.

\section{ACKNOWLEDGEMENTS}

The author would like to thank respondents and owners of the surveyed private health facilities, who voluntarily took part in this study. I am thankful for USAID - Private Health Sector Program for funding the fieldwork. I would like to thank Derebe, Tadesse Abate who assisted me with the map illustration. I am indebted to all data collectors and supervisors for their quality of work. The finding and opinions expressed by the author may not reflect the views of the employing or funding organizations.

\section{REFERENCES}

[1] Ethiopian Health and Nutrition Research Institute: Malaria Laboratory Diagnosis External Quality Assessment Scheme Guidelines. Federal Democratic Republic of Ethiopia Ministry of Health, Addis Ababa, 2009.

[2] World Health Organization (WHO): WHO Global Malaria Program. World Malaria Report 2013. WHO, Geneva, 2014.

[3] World Health Organization (WHO). 2010. Guidelines for the treatment of malaria. $2^{\text {nd }}$ edition.WHO, Geneva, 2010. From: http://www. who.int/malaria/publications/atoz/97892415479 25/en/index.html (accessed 17 August 2015)

[4] Federal Democratic Republic of Ethiopia Ministry of Health: Malaria guidelines: Diagnosis and Treatment Guidelines for Health Workers in Ethiopia. ( $3^{\text {rd }}$ ed). FDREMOH, Addis Ababa, 2012.

[5] Federal Democratic Republic of Ethiopia Ministry of Health: Ethiopia's Fifth National Health Accounts 2010/2011. Addis Ababa, Ethiopia, 2014.

[6] Jima D, Getachew A, Bilak H, Steketee RW, Emerson PM, Graves PM, Gebre T, Reithinger $\mathrm{R}$, Hwang $\mathrm{J}$, the Ethiopian Malaria Indicator Survey Working Group: Malaria Indicator Survey 2007, Ethiopia: Coverage and use of major malaria prevention and control interventions. Malaria J. 2010, 9:1-12.

[7] Deressa W, and Ali A: Malaria-related perception and practices of women with children under the age of five years in rural Ethiopia.BMC Public Health. 2009, 9:259.

[8] Brugha R, \&Zwi A: Improving the quality of private sector delivery of public health services: challenges and strategies. Health policy and planning, 1998; 13(2), 107-120.

[9] Khin HS, Chen I, White C, Sudhinaraset M, McFarland W, Littrell M, Mopvaiu D, \&Aung T: (2015). Availability and quality of antimalarials among private sector outlets in Myanmar in 2012: results from a large, community- based, cross-sectional survey before a large-scale intervention. Malaria journal, 2015; 14(1): 269.

[10]World Health Organization (WHO): Partnerships for malaria control: Engaging the formal and informal private sector. WHO, Geneva, 2006. 
[11] World Health Organization (2007). Everybody's business: strengthening health systems to improve health outcomes: WHO's framework for action. Geneva: World Health Organization.

[12] Federal Ministry of Health Ethiopia (FMOH): National Malaria Strategic Plan (2014-2020). Addis Ababa: FMOH, 2014.

[13]Emmett W, Whalen C. Final Evaluation: The Private Sector Program in Ethiopia: Assessing the Foundation for a Continuum Focused on Public-Private Health Sector Collaboration in addressing HIV/AIDS and Tuberculosis in Ethiopia. Washington, DC: Global Health Technical Assistance Project; 2008.

[14]Rao P, Gabre-Kidan T, Mubangizi DB, \&Sulzbach S: Leveraging the private health sector to enhance HIV service delivery in lowerincome countries. JAIDS Journal of Acquired Immune Deficiency Syndromes, 2011; 57, S116S119.

[15]Gilbert K, Poer K, Andualem W, Zaid T, \&Alemayehu A: Assessment of Readiness of Private Higher Clinics in Addis Ababa, Ethiopia, to Initiate ART Services. Bethesda, MD: Private Sector Program-Ethiopia, Abt Associates Inc.2008.

[16] Kassu A \&Aseffa A (1999) Laboratory services in health centers within Amhara region, north Ethiopia. East African Medical Journal 76, 239242.

[17]Hailegiorgis B, Girma S, Melaku Z, Teshi T, Demeke L, Gebresellasie S, Yadeta D, Tibesso G, Whitehurst N, Yamo E, Carter J, \&Reithinger $\mathrm{R}$ : Laboratory malaria diagnostic capacity in health facilities in five administrative zones of Oromia Regional State, Ethiopia. Tropical Medicine \& International Health, 2010; 15(12): 1449-1457.

[18] Abreha T, Alemayehu B, Tadesse Y, Gebresillassie S, Tadesse A, Demeke L, Zewde T, Habtamu M, Tadesse M, Yadeta D, TeshomeD, Mekasha A, Gobena K, Bogale H, Melaku Z, Reithinger R, \&Teka H: Malaria diagnostic capacity in health facilities in Ethiopia. Malar J, 2014; 13, 292.

[19] Ekawati L L, Herdiana H, Sumiwi ME, Barussanah C, Ainun C, Sabri S, Maulana T, Rahmadyani R, Maneh R, Yani M, Valenti P, Elyazar IRF \& Hawley WA: A comprehensive assessment of the malaria microscopy system of Aceh, Indonesia, in preparation for malaria elimination. Malaria Journal 2015; 14(1), 240.

[20] World Health Organization (WHO): Malaria microscopy quality assurance manual. World Health Organization, 2009.

[21]Federal Democratic Republic of Ethiopia, Ministry of Health: Malaria: Diagnosis and Treatment Guidelines for Health Workers in Ethiopia. ( $2^{\text {nd }}$ ed). FDREMOH, Addis Ababa, 2004.

[22] Jerene D, Fentie G, Teka M, Girma S, Chibsa S, Teka H, \&Reithinger R: The role of private health facilities in the provision of malaria case management and prevention services in four zones of Oromia Regional State, Ethiopia. International Health.2012, 4:70-73.

[23] National Bureau of Statistics Tanzania and Macro International Inc.: Tanzania Service Provision Assessment Survey 2006. National Bureau of Statistics and Macro International Inc., Dar-es-Salaam, 2007.

[24] Amexo M, Tolhurst R, Barnish G, Bates I: Malaria misdiagnosis: effects on the poor and vulnerable. Lancet. 2004,364:1896-1898.

[25] Achan J, Tibenderana J, Kyabayinze D, Mawejje H, Mugizi R, Mpeka B, Ralisuna A, D'Alessansro U: Case management of severe malaria - A forgotten practice: experiences from health facilities in Uganda. PLoS ONE. 2011, 6:e17053.

[26] Argaw MD: Knowledge and practice on malaria diagnosis and treatment among healthcare providers working in private health facilities in Ethiopia. Malaria World Journal 2015;6(10):18 .

Citation: Mesele DamteArgaw. Malaria Diagnostic Capacity of Private Health Facilities in Ethiopia. ARC Journal of Public Health and Community Medicine.2017; 2(4):18-27: dx.doi.org/10.20431/2456-0596. 0204004

Copyright: (0) 2017 Authors. This is an open-access article distributed under the terms of the Creative Commons Attribution License, which permits unrestricted use, distribution, and reproduction in any medium, provided the original author and source are credited. 